\title{
Negative Matter as Unified Dark Matter and Dark Energy: Simplest Model, Theory and Nine Tests
}

\author{
Yi-Fang Chang \\ Department of Physics, Yunnan University, Kunming, 650091, China \\ Email: yifangch@sina.com
}

Received Nov 2020

Received in revised: Dec 2020

Published: Dec 2020

\begin{abstract}
Based on Dirac's negative energy, we propose and study the negative matter. Bondi's results are wrong. First, the negative matter can be the simplest model of unified dark matter and dark energy. Next, we discuss various possible theories of the negative matter: some field equations, similar electrodynamics, field equations with non-symmetry, etc. Third, the quantum theory of negative matter is researched. Matter surrounded by dark-negative matter corresponds to an infinitely deep potential trap in quantum mechanics and forms a base of the universal wave-particle duality and quantum mechanics. Fourth, we propose the mechanism of inflation as the origin of positive-negative matters created from nothing. Fifth, assume that dark matter is completely the negative matter, and we may calculate an evolutional ratio between total matter and usual matter from 1 of inflation and the radiation-dominated universe to 7.88 of the present matter-dominated universe. It agrees with the observed value 6.36 7. Sixth, we research the relativity of the negative matter and theory in Lobachevskian geometry. Seventh, we propose a judgment test of the negative matter as dark matter is opposite repulsive lensing and other eight possible tests. Eighty, we propose a figure on the unification of the four basic interactions in three-dimensional space, in which the "running" coupling constants of strong and weak interactions transform each other. The negative matter as a candidate of unification of dark matter and dark energy is not only the simplest, and may explain inflation and be calculated and tested.
\end{abstract}

Keywords: Negative Matter, Repulsion, Dark Matter, Dark Energy, Inflation, Quantum, Ratio, Test, Field, Equation.

C2020 The Authors. Published by Fundamental Journals. This is an open access article under the CC BY-NC https://creativecommons.org/licenses/by-nc/4.0/

https://doi.org/10.14331/ijfps.2020.330141

\section{INTRODUCTION}

Now investigation of dark matter and dark energy is a basic problem and focus in astronomy, astrophysics, cosmology and total physics. General hypothesis is that dark matter and dark energy are two different concepts. The dark matter in the Galaxy, in group of galaxies and cluster of galaxies, in the universe, is confirmed by the mass-to-light ratio and the galactic rotational curves, etc. (Binney \& Tremaine, 2011; Kahn \& Woltjer, 1959; Zwicky, 1937).

The dark matter seems to have mass and may become huge conglomeration. Cosmologists compute that the gravitational 
conglomeration of these dark matters is a key function for the process formed galaxies from general matter.

The dark matter is possibly the weakly interacting massive particles (WIMP), the models of dark matter include neutrino with mass, baryonic dark matter and nonbaryonic dark matter (Copi, Schramm, \& Turner, 1995; Gerhard \& Silk, 1996), monopole, supersymmetric dark matter (Jungman, Kamionkowski, \& Griest, 1996), axion (Shellard \& Battye, 1998), supersymmetric neutralino, KK particle (Hooper \& Profumo, 2007), etc., or due to modification of Newtonian dynamics (MOND). Cosmologist divides the candidate of the dark matter into three types: hot, warm and cold dark matter. Chevallier and Polarski (2001), searched accelerating universes with scaling dark matter. Clowe et al. (2006), discussed directly an empirical proof of the existence of dark matter.

The simplest kind of dark matter model is to add phenomenally a real scalar field $\varphi$ as the dark matter field in the standard model (Barger, Langacker, McCaskey, Ramsey-Musolf, \& Shaughnessy, 2008; McDonald, 1994). Dark matter particles have typical characteristics of WIMP, and can have clear theoretical predictions. So far, small mass dark matter has almost been ruled out by experiments. Cold dark matters are the nonrelativistic particles.

The dark energy as a huge repulsive force is proposed in order to explain the accelerating Universe discovered from observes of supernovae (Peebles \& Ratra, 2003; Perlmutter et al., 1998; Riess et al., 1998), and may unify many different results of observations. The dark energy seems to be the energy of vacuum, and be zero mass. It distributes uniformly in the whole space, and its interactions are repulsive. Usually assume that the dark energy connects with the cosmological constant $\Lambda$ (Weinberg, 1989), and is a dynamical scalar field (quintessence model) in which the pressure-density ratio $w>$ $-1, w<-1$ for phantom model, in Quintom model (The Quintom scenario is a hypothetical model of dark energy) evolution can pass through $w=-1$. When $w=0$, universe moderates, $w=-1 / 3$ does not accelerate or decelerate, only $w<-1 / 3$ can accelerate expansion.

Dark energy in the dynamic scalar field exists usually evolution. Dark energy as the modified general relativity can explain many effects of the dark energy, but cannot explain the dark matter. In the parameterization of dark energy, 8 basic parameters for density are too much.

Mortonson, Hu, and Huterer (2010), predicted the testable dark energy from current data. Scherrer (2004), proposed a new kessence models in which the Lagrangian $p$ is a function only of the derivatives of a scalar field. In the model the universe fills a kind of invisible fluid, and the models can serve as a unified model for dark matter and dark energy. Soleng (1995), discussed dark matter and non-Newtonian gravity from general relativity coupled to a fluid of strings. But, the tests of some known theories are very difficult.

In the universe the dark matter has about $24 \%$ and the dark energy has about $72 \%$, only $4 \%$ is visible matter. For new data, usual matter is $4.84 \%$, total matter is $30.8 \%$, and dark energy is $69.2 \%$ (Adam et al., 2016; Tanabashi et al., 2018).

Recently, Elahi and Khatibi (2019) discussed multicomponent dark matter in a non-Abelian dark sector. Kim, Lane, Lee, Lewis, and Sullivan (2020), searched dark photons with maverick top partners.
It is well-known that in 1928 Dirac predicted anti-particles and the negative energy state from his equation, and he emphasized: "we cannot ignore the negative energy states" (P. A. M. Dirac, 1930).

In order to prevent to jump continuously from positive energy state to negative energy state in the quantum theories, and keep the stability of world, Dirac proposed that as long as suppose that all the states of negative energy are occupied except perhaps a few of small velocity. The vacuum of the realistic world has already been filling with all negative energy state, such the Pauli Exclusion Principle will come into play and prevent more than one electron going into any one state, and avoid this jumping difficulty.

It is namely the well-known Dirac negative energy sea and whose vacancy or hole is an anti-particle (or opposite particle). From this the annihilation and creation between positive and opposite particles may be predicted. There is exact description in The Principles of Quantum Mechanics (P. Dirac, 1958).

But, it prevent only jump of fermions, but cannot be applied to bosons. Therefore, the stability problem exists still. In fact, the negative energy state appears in all relativity theories as $E= \pm \sqrt{p^{2} c^{2}+m^{2} c^{4}}$, even also in the classical theory. Moreover, according to the gravitational force,

$$
F=-\frac{G}{r^{2}} m_{1} m_{2}
$$

its potential energy $V=-\left(G m_{1} m_{2}\right) / r$ is negative, and mass is $m_{V}=-\left(G m_{1} m_{2}\right) / r c^{2}$. We proposed the negative matter developed the Dirac negative energy state may unify dark matter and dark energy (Chang, 2007b, 2011, 2013b, 2014a, 2017, 2019).

We think the anti-(opposite) matter and the negative matter are different. The anti-matter is that some properties of matter are opposite, for instance, charge, baryon number, lepton number, strangeness number and so on, but their masses and total energy are still positive. These particles include positron and various anti-particles. The existence of these particles is already verified. Both positive and opposite matters meet to annihilate to photons with conservation of energy and zerocharge. The negative matter has a negative mass and total energy. The creation of negative matter is difficult, but its existence will be stable.

For negative mass Bondi (1957) proposed three kinds of mass: inertial, passive gravitational, and active gravitational mass, and there are four cases. But, this is a question: Bondi believes that the positive body will attract the negative one (since all bodies are attracted by it), etc. It is a fallacy with contradictions. According to the principle of equivalence in general relativity, inertial mass and gravitational mass must be equal always.

Therefore, there are only three cases of interactions: positive and positive matters, positive and negative matters, negative and negative matters (Chang, 2007b, 2011).

In this paper, we research various theories of the negative matter as unified dark matter and dark energy, and propose the mechanism of inflation, and nine tests on existence of negative matter. 
NEGATIVE MATTER IS THE SIMPLEST MODEL OF UNIFIED DARK MATTER AND DARK ENERGY

Based on Dirac's negative energy state, we proposed the negative mass, and a basic axiom (the no-contradiction of natural laws) and two foundational principles:

1. The negative matter obeys the same natural laws of usual matter, including classical, relativistic and quantum physics.

2. There is usual gravitational force between negative-negative matters, but it is universal repulsive force between the positive and negative matters based on the general formula (1) (Chang, 2011). Such the positive and negative matters are two regions of topological separation in general case by different interactions (Fig.1), so the negative matter is invisible. This is the simplest candidate of dark matter, and can be unified dark energy due to the repulsive force between the positive and negative matters. It may explain their some phenomena of dark matter and dark (Chang, 2007b, 2011, 2013b, 2014a, 2017, 2019). O is a balance point between gravitation and repulsion, and may form a balance equipotential surface in space-time. The negative matter should be a necessary development of Dirac theory. Fig. 1 may be simulated by two repulsive magnets.

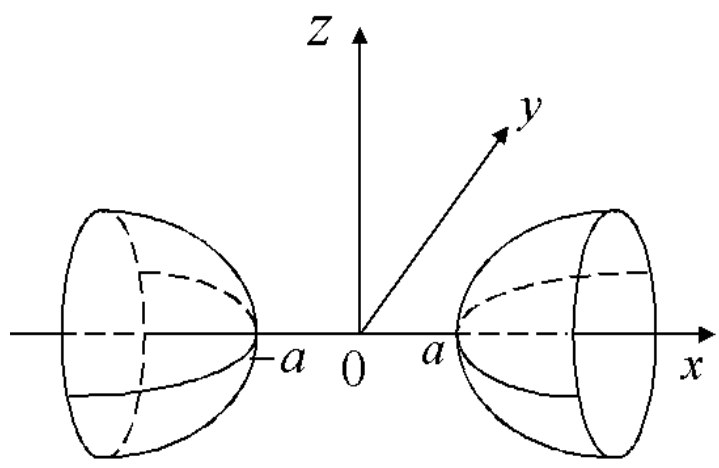

Fig. 1 The hyperboloid of two sheets

We researched the field equations of repulsive force between positive-negative matters. The field equations of general relativity on the negative matter are,

$$
G_{\mu v}=8 \pi k\left(T_{\mu v}-T_{\mu \nu}^{\prime}\right),
$$

i.e.,

$$
G_{\mu v}+8 \pi k T_{\mu v}^{\prime}=8 \pi k T_{\mu v}=G_{\mu v}-\Lambda g_{\mu v}
$$

So the cosmological constant $\Lambda$ corresponds to the negative matter, and is

$$
\Lambda=-8 \pi k T_{\mu \nu}^{\prime} / g_{\mu v}=-\left[\rho^{\prime}+\left(p^{\prime} / c^{2}\right)\right]\left(u_{\mu} u_{v} / g_{\mu v}\right)+p^{\prime}
$$

On the other hand, the gravitational field equation with the cosmological constant is extended to

$$
G_{\mu v}=8 \pi k T_{\mu v} \Rightarrow 8 \pi k\left(T_{\mu v}+\Lambda g_{\mu v}\right)
$$

Here $\Lambda g_{\mu v}$ corresponds to the negative energy state and vacuum energy, i.e., Dirac Sea.

The negative matter determines the cosmological constant, and is consistent with conformal gravity theory (Mannheim, 1992). The Friedmann equation is

$$
\ddot{R}(t)=-\frac{4}{3} \pi G\left[\left(\rho_{1}-\rho_{2}\right)+3\left(p_{1}-p_{2}\right) / c^{2}\right] R(t)
$$

in which $\left(\rho_{1}-\rho_{2}\right)+3\left(p_{1}-p_{2}\right) / c^{2}$ is effective mass density.

$$
\dot{R}^{2}-\frac{8}{3} \pi G\left(\rho_{1}-\rho_{2}\right) R^{2}=2 C
$$

in which $\dot{R}\left(t_{0}\right)=H_{0}$ is the Hubble constant. The density parameter is

$$
\Omega_{0}=\frac{8 \pi G \rho_{0}}{3 H_{0}^{2}}=\frac{\rho_{0}}{\rho_{c}}, \rho_{0}=\rho_{1}-\rho_{2}
$$

It is an observed density. The accelerating expansion of the universe shows

$$
\left(\rho_{1}-\rho_{2}\right)+3\left(p_{1}-p_{2}\right) / c^{2}<0
$$

i.e.,

$$
\rho_{2}+\left(3 p_{2} / c^{2}\right)>\rho_{1}+\left(3 p_{1} / c^{2}\right)
$$

The negative matter is more than the positive matter. But, the negative matter cannot yet explain the apparent increase of mass due to dark matter.

Caldwell (2002), proposed phantom as cosmological consequences of a dark energy component with super-negative equation of state, whose cosmic energy density has negative pressure. Then phantom becomes an important dark energy model. Piao and Zhang (2004), researched phantom inflation and primordial perturbation spectrum. Hong, Lee, Lee, and Oh (2008), considered a higher dimensional gravity theory with a negative kinetic energy phantom field and a cosmological constant. Scherrer and Sen (2008) examined phantom dark energy models produced by a field with a negative kinetic term. Chimento, Forte, Lazkoz, and Richarte (2009), discussed the dark energy density derived from the 3 -scalar phantom field, and its negative component plays the role of the negative part of a classical Dirac field. Gonzalez and Guzman (2009), presented the full nonlinear study of a phantom scalar field accreted into a black hole, which includes that the total energy of the space-time is positive or negative. The total energy is negative, so it should be namely a type of negative matter. Feng, Li, and Saridakis (2010), researched preventing eternality in phantom inflation. Saridakis and Sushkov (2010), discussed quintessence and phantom cosmology with nonminimal derivative coupling.

P. Dirac (1958), pointed out: The physical laws are symmetrical between the positive and negative charge. Further, the physical laws should be also symmetrical between the positive and negative matters. It forms just the most perfect symmetrical world that four matters on positive, opposite, and 
negative, negative-opposite particles exist together (Chang, 2016, 2017). If the negative matter is verified, a new and complete world will be exhibited (Fig. 2).

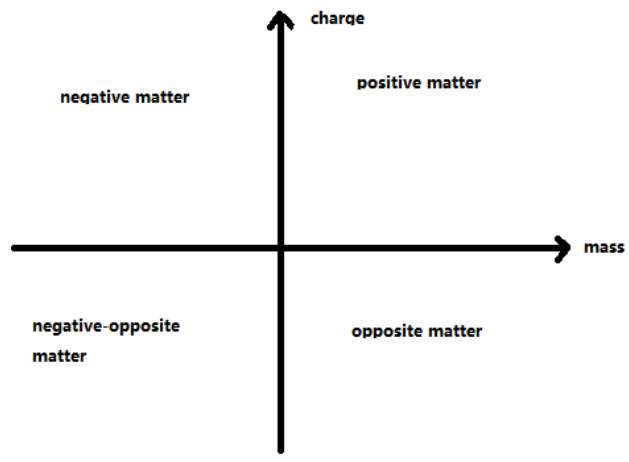

Fig. 2. A new most perfect symmetrical world

In the complete world, between positive matters is gravity and corresponding graviton, between the negative matters is also gravity, which is the same graviton? Between positive and the negative matters is repulsion, which should correspond repulsion that is anti-graviton, $J=-2$. But, current electromagnetic interaction, both gravity and repulsion correspond to the same photon, such it is also possible that graviton and repulsion are the same.

\section{THE MOST COMPLETE THEORY OF NEGATIVE MATTER}

A main character of the negative matter is completely the same with various known theories. Because space-time is the same, kinematics of the negative matter should be the same. But, their dynamics are very different. Assuming that the known formula is the same, the $p=m v<0, F=m a<0$.

If formula is different and mass is the same, it will be $F=-m a, F=-(-m) a>0$.

For the negative matter, the Lagrangian of nonrelativistic particle in field is (L. D. Landau \& Lifshitz, 1971),

$$
L=m c^{2}-\frac{1}{2} m v^{2}+m \phi
$$

The corresponding action function is

$$
\begin{gathered}
S=\int L d t=m c \int\left(c d t-\frac{1}{2} \frac{v}{c} d r+\frac{1}{c} \phi d t\right) \\
d s=-c d t+\frac{1}{2} \frac{v}{c} d r-\frac{1}{c} \phi d t \\
d s^{2} \approx\left(c^{2}+2 \phi\right) d t^{2}-d r^{2}\left(1+\frac{1}{c^{2}} \phi\right)
\end{gathered}
$$

Therefore,

$$
g_{00}=-1-\frac{2}{c^{2}} \phi, g_{r r}=1+\frac{1}{c^{2}} \phi
$$

For the negative matter and the field between positive-negative matters, their difference is only the potential $\varphi$. According to the correspondence principle it changes to repulsion.

$$
R_{i}^{k}=\frac{8 \pi k}{c^{4}}\left(T_{i}^{k}-\frac{1}{2} \delta_{i}^{k} T\right)
$$

Where here $T_{0}^{0}=\rho_{-} c^{2}$.
Let $i=k=0$,

$$
\begin{gathered}
R_{0}^{0}=\frac{4 \pi k}{c^{4}} T_{0}^{0}=\frac{4 \pi k}{c^{2}} \rho_{-}>0, \quad \text { (for negative matter) } \\
R_{0}^{0}=-\frac{4 \pi k}{c^{2}} \rho_{+}<0 \quad \text { (for positive matter) } \\
R_{00}=-R_{0}^{0}=\frac{\partial \Gamma_{00}^{\alpha}}{\partial x^{\alpha}}, \Gamma_{00}^{\alpha} \cong-\frac{1}{2} g^{\alpha \beta} \frac{\partial g_{00}}{\partial x^{\beta}}=\frac{1}{c^{2}} \frac{\partial \phi}{\partial x^{\alpha}} \\
R_{0}^{0}=-\frac{\partial \Gamma_{00}^{\alpha}}{\partial x^{\alpha}}=-\frac{1}{c^{2}} \frac{\partial^{2} \phi}{\partial x^{\alpha 2}}=-\frac{1}{c^{2}} \Delta \phi \\
\Delta \phi=-4 \pi k \rho_{-}<0
\end{gathered}
$$

The potential of the negative matter is

$$
\begin{gathered}
\phi_{-}=\frac{G M_{-}}{r} \\
F=-\frac{d V}{d r}=-\frac{d}{d r}\left( \pm m_{ \pm} \phi_{-}\right)= \pm \frac{G m_{ \pm} M_{-}}{r}
\end{gathered}
$$

For positive matter $F>0$ is repulsion. For the negative matter $F<0$ is gravitation. We assume that positive matter and negative matter are respectively the same gravitation fields and corresponding field equations only with different positive or negative mass (Chang, 2007b, 2013b), which include Schwarzshild solution, and spherical symmetry, etc.

Such conclusion is that universe includes three parts, whose fields may be the scalar field similar to Newton theory, or the tensor field similar to general relativity, or the vector field similar to the electrodynamics,

I. The positive matters are gravitation and Riemannian geometry.

II. The negative matters are gravitation and Riemannian geometry. Riemannian geometry corresponds to closed universe.

III. Between the positive and negative matters are repulsion, which is probably Lobachevskian geometry, i.e., the hyperbolic geometry. This corresponds to open universe, and derived cosmic accelerated expansion. The repulsion field and field equations have not the spherical symmetry. Both geometries may be unified by general differential geometry.

Further, it should include the total field and its equation of these three fields. This is not only unified of gravitational field and electromagnetic field (Chang, 1989, 2014b), and is unifying four matters (Chang, 2016, 2017, 2020).

For III case, we discuss some possible developments. Assume that the relations between positive-negative matters are probably an analogous field with two kinds of charge.

1) Similar static electricity $F=e E \rightarrow F= \pm m E$ ( $\mathrm{E}$ is force intensity) $=m a, a= \pm E$. Mass is similar charge, but the biggest difference lies the same mass gravity and opposite mass repulsion each other for positive and negative matters. A similar static electrical field is namely a scalar field. 
2) From this derives similar magnetic line of force, and develop similar magnetism. The generalized momentum is ( $\mathrm{L}$. D. Landau \& Lifshitz, 1971)

$$
\begin{gathered}
P=m_{ \pm}(v+A / c) \\
F=m_{ \pm}\left(E+\frac{v}{c} \times B\right)=m_{ \pm} a
\end{gathered}
$$

Here $\mathrm{A}$ is a vector potential. The negative matter is similar to monopole, whose topological solution just corresponds to positive and negative matters. It is a huge monopole.

3) It is known that general relativity derived already a similar magnetic field and similar Lorentz force and equation (Einstein, 1955; L. D. Landau \& Lifshitz, 1971).

4) The repulsion field and field equations between positivenegative matters are similar electromagnetic field. It may obtain similar electrodynamics and Maxwell equations, even electromagnetic general relativity (Chang, 2005).

5) This may develop similar quantum electrodynamics (QED). Field equations possibly are related to metric $g_{\mu v}$ symmetry or anti-symmetry. Positive matter and negative matter are all fields with symmetrical $g_{\mu \nu}$, etc; field between positive and negative matter is anti-symmetrical $g_{\mu v}$, etc. It is probably related to torsion.

The torsion tensor is $\Omega_{i j}^{k}=\Gamma_{i j}^{k}-\Gamma_{j i}^{k}$, i.e., the $\Gamma_{i j}^{k}$ have torsion for non-symmetric $i, j$. From this derives the drag effects between the negative matter and positive-negative matters. Their "gravitational wave" should have difference. Further, they are $R_{i j k}^{l}$ symmetry or anti-symmetry. For the nonsymmetric field Einstein (1955) discussed $A=\frac{1}{1+c r^{2}}$ in cosmology, here $c>0$ is a sphere space, and $c<0$ is a pseudosphere space. This is similar to the unified theories of gravitational and electromagnetic fields (Chang, 1989, 2014b), or the unified general relativity of gravitational and electromagnetic fields (Chang, 2005). The geodesic equations of two geometries are the same form,

$$
\frac{d^{2} x^{\mu}}{d s^{2}}+\Gamma_{\alpha \beta}^{\mu} \frac{d x^{\alpha}}{d s} \frac{d x^{\beta}}{d s}=0
$$

But, $\Gamma_{\alpha \beta}^{\mu}$ have difference. From (26) the geodesics depend only on the symmetric part $\Gamma_{(\alpha \beta)}^{\mu}=\Gamma_{\alpha \beta}^{\mu}+\Gamma_{\beta \alpha}^{\mu}$ of the connation. The anti-symmetric part of $\Gamma_{\alpha \beta}^{\mu}$ is possibly related to other quantity, for example, torsion.

$$
\frac{d R}{d t}=\frac{4}{3} \pi G a t=H t
$$

Einstein field equation under Friedmann-Robertson-Walker metric is:
Pure time component,

$$
\ddot{R}=-\frac{4 \pi G}{3}\left(\rho+\frac{3 p}{c^{2}}\right) R
$$

Pure spatial component,

$$
R \ddot{R}+2 \dot{R}^{2}+2 k=4 \pi G(\rho-p) R^{2}
$$

This is the acceleration of the cosmic expansion rate. Both mergers are

$$
\dot{R}^{2}+k=\frac{8}{3} \pi G \rho R^{2}
$$

For the negative matter density and pressure all are negative, or only density is negative.

$$
\begin{gathered}
\ddot{R}=-\frac{4 \pi G}{3}\left[\left(\rho_{+}-\rho_{-}\right)+\frac{3\left(p_{+} \pm p_{-}\right)}{c^{2}}\right] R \\
R \ddot{R}+2 \dot{R}^{2}+2 k=4 \pi G\left[\left(\rho_{+}-\rho_{-}\right)-\left(p_{+}-p_{+}\right)\right] R^{2}
\end{gathered}
$$

Both mergers are

$$
\dot{R}^{2}+k=\frac{8}{3} \pi G\left(\rho_{+}-\rho_{-}\right) R^{2}
$$

Here

$$
a=\left(\rho_{-}-\rho_{+}\right)-\frac{3\left(p_{+} \pm p_{-}\right)}{c^{2}} .
$$

When

$$
\rho_{-}>\rho_{+}+\frac{3\left(p_{+} \pm p_{-}\right)}{c^{2}}
$$

is repulsion, it is accelerate expansion. The energy-momentum tensor for macroscopic bodies is (L. Landau \& Lifshitz, 1977),

$$
\begin{gathered}
T^{i k}=\left(p+\rho c^{2}\right) u^{i} u^{k}-p g^{i k} \\
T_{i}^{i}=\rho c^{2}-3 p
\end{gathered}
$$

An inequality is always valid for the pressure and density of a macroscopic body

$$
\rho>\frac{3 p}{c^{2}}
$$

Its limit is $\rho=\frac{3 p}{c^{2}}$ for electromagnetic interaction, which corresponds to radiation-dominated universe.

When $\rho_{-}>\rho_{+}+\frac{3\left(p_{+} \pm p_{-}\right)}{c^{2}}$ is repulsion, and the cosmic accelerated is expansion.

Assume that $p_{+}=p_{-}=p$, so $\rho_{-}>\rho_{+}$or $\rho_{-}>\rho_{+}+\frac{6 p}{c^{2}}$. 
If $\rho_{-}=7 \rho_{+}=7 \rho$, so $\rho>\frac{p}{c^{2}}$ is repulsion. In a word, these cases are all repulsion. The universe as a relative particle or radiation $\rho_{-}= \pm 3 p_{-}= \pm 3 p . \rho_{-}>\rho_{+}+6 p=3 \rho_{+}$, and there have more negative matters. It corresponds to dark matter is $24 \%(30.8 \%)$ and usual matter is $4 \%(4.84 \%)$, and $24 / 4=6$ (30.8/4.84=6.36). The universe as the non-relativistic loose matter $p=0$, i.e., $\rho_{-}>\rho_{+}$. When the cosmological term exists,

$$
\ddot{R}=-\frac{4 \pi G}{3}\left(\rho_{+}-\rho_{-}\right) R+\frac{\Lambda}{3} R
$$

i.e.

$$
\Lambda=4 \pi G \rho_{-}>0, \quad \text { or } \quad \Lambda=4 \pi G\left(\rho_{-} \mp 3 p_{-} / c^{2}\right)
$$

Let $a=\left(\rho_{-}-\rho_{+}\right)-\frac{3\left(p_{+} \pm p_{-}\right)}{c^{2}}$, for Eq.(31) integral once

$$
\frac{d R}{d t}=\frac{4}{3} \pi G a t=H t
$$

Integral again $R=R_{0} e^{H t}$ is namely inflation model for $a>0$. The end of inflation may produce the primordial gravitational wave determined by gravitational interaction.

We proposed a test of the negative matter (Chang, 2011): When the move speed between positive and negative matters is very big, and the kinetic energy is bigger than the potential energy, their colliding result will be a complete annihilation, which will produces huge gravitational (or similar repulsive) wave.

We proved the gravitational wave is nonlinear wave, and the velocity of the gravitational wave should be different with the velocity of light, at least since light deflects while the gravitational wave propagates along a straight line in a strong gravitational field (Chang, 1996). Now the gravitational wave has been confirmed by LIGO and Virgo (Abbott et al., 2016a, 2016b). We forecasted that further investigations may discover difference between gravitational wave and electromagnetic wave (Chang, 2016). In 2017 detectors observed association with the $\gamma$-ray burst (GRB) $1.7 \mathrm{~s}$ after GW170817 (Abbott et al., 2017).

New observed gravitational waves proved they are nonlinear waves, not only collision of two black holes or binary neutron star (BNS) must be nonlinear mechanism, and released huge energy and the observed signals of gravitational waves are necessarily pulse wave. We calculate to obtain total delay time is $0.1792 \mathrm{~s}$ for BNS by gravitational redshift and the deflection of light. It is about $1 / 10$ of observed delay time $1.7 \mathrm{~s}$. The gravitational wave and electromagnetic wave pass through the luminosity distance about 40 megaparsecs (about 130 million light years) and many gravitational redshifts and deflections, it is possible that both difference increases 10 times (Chang, 2018a)

\section{NEGATIVE MATTER AND QUANTUM THEORY}

In the quantum mechanics the negative matter may be still $E=h v$, in which $E \rightarrow-E$ and $-h \rightarrow h$ (Chang, 2011, 2013b). Klein-Gordon equation is the same since $m^{2}$ term may corresponds to $\pm m$, both describe bosons. But all bosons with mass are unstable. We must obtain Dirac equations of negative matter, which are only $m \rightarrow-m$ (Chang, 2014a),

$$
\left(\gamma_{\mu} \partial_{\mu}-m\right) \psi=0
$$

Dirac equations may derive two solutions of $m>0$ or $m<0$, which is Dirac's negative energy state. Fermions are stable, and correspond to dark matter. Schrödinger equation is,

$$
i \hbar \frac{\partial \psi}{\partial t}=\frac{\hbar^{2}}{2 m} \nabla^{2} \psi-U(r) \psi
$$

Here only $U \rightarrow-U$. Matter surrounded by dark-negative matter at a certain direction corresponds to the extensive quantum mechanics (here $\hbar \rightarrow H$ ) (Chang, 1990, 2002, 2018b) with an infinite deep potential trap.

Its energy spectrum and momentum of Schrödinger equation is necessarily discrete (Ballentine, 1998; Flügge, 2012; L. Landau \& Lifshitz, 1977),

$$
E_{n}=\frac{H^{2} \pi^{2}}{2 M R^{2}} n^{2} \quad, \quad p_{n}= \pm \frac{H \pi}{R} n
$$

Its cosmic wave function is

$$
\psi_{n}(r)=A_{n} \sin \left(\frac{n \pi}{R} r\right)
$$

Both form just a base of the universal wave-particle duality and total general quantum mechanics. The corresponding onedimensional density is

$$
\rho\left(E_{n}\right)=\frac{R}{2 \pi H} \sqrt{\frac{2 M}{E_{n}}}
$$

two-dimensional density is

$$
\rho\left(E_{n}\right)=\frac{R^{2}}{2 \pi H^{2}} M
$$

three-dimensional density is

$$
\rho\left(E_{n}\right)=\frac{R^{3}(2 M)^{3 / 2}}{4 \pi^{2} H^{3}} \sqrt{E_{n}}
$$

It may be similar QED, which constantly produce positive and negative matters, then annihilate under very big field.

By a similar Higgs mechanism the positive and negative matters may be produced at the same time. Introducing scalar fields is namely Higgs mechanism.

$$
\begin{gathered}
L=\frac{1}{2} \partial_{\mu} \phi \partial^{\mu} \phi-\frac{1}{2} \mu^{2} \phi^{2}-\frac{1}{4} \lambda \phi^{4} \\
V=\frac{1}{2} \mu^{2} \phi^{2}+\frac{1}{4} \lambda \phi^{4}
\end{gathered}
$$

Minimum ground state is 


$$
\begin{aligned}
& \frac{d V}{d \phi}=\left(\mu^{2}+\lambda \phi^{2}\right) \phi=0 \\
& \phi=0 \text { or } \phi= \pm \sqrt{-\frac{\mu^{2}}{\lambda}}
\end{aligned}
$$

\section{NEGATIVE MATTER AS MECHANISM OF INFLATION}

In cosmology it is an important progress that (Guth, 1981) first proposed inflation whose time origin is from $10^{-32} \mathrm{~s}$, and cosmic scale factor exponential expansion $a(t) \approx e^{H t}$. The universe has expanded at least $10^{26}$ times. Inflation explains the flatness and horizon, etc., problems. But, it has many questions, for example, the inflation origin is near absolute zero temperature, and the vacuum energy is almost zero, etc. It must be very high energy. Next (A. D. Linde, 1982, 1983) and Albrecht and Steinhardt (1982) proposed the chaotic inflation. The universe first was inflation, and slowed down 11 billion years ago. Then it has been be accelerated expansion for 5 billion years.

S. Hawking, N. Turok and J. Hartle used instanton to replace singularity of cosmological origin. Lucchin and Matarrese (1985), discussed power law inflation. La and Steinhardt (1989), proposed the extended inflation. A. Linde (1994), developed the hybrid inflation. Dodelson, Kinney, and Kolb (1997), searched classification of various inflation models. Russo (2004), obtained exact solution of scalar field cosmology with exponential potentials and transient acceleration.

The inflation model is a new result of the combination of modern quantum field theory and cosmology, and it is a big development of very early cosmology. Is should have a reasonable theoretical origin in order to produce enough inflation. But, so far this has not a reasonable mechanism of inflation, and only introduces phenomenally a scalar field.

We proposed that the mechanism of inflation cosmology due to a huge repulsive force between the positive matter and negative matter created at the same time in quantum fluctuations. This corresponds to the cosmological mode created from nothing to all things from vacuum (Chang, 2007b, 2013b). In 1963 Dirac supposed that an important direction of developing science is study of vacuum. It is known that Klein-Gordon (KG) equation of the scalar field is

$$
\left(\nabla^{2}-m^{2}\right) \phi=G
$$

Its solution is

$$
\phi=-G e^{-m r} / r
$$

Chaotic inflation phenomenally introduces a scalar field, which is similar to the strong repulsion field at very small scale. It produces quantum effect, and the interaction between positive-negative matters is nonlinear, which may obtain chaos. Different universes are positive and negative matters produce bubbles. This may form the parallel worlds, or the many-worlds, or multiverse, etc. It can combine Linde chaotic inflation, but both differences are different scales and times.
Inflation is related to Planck time-space. It is origin of inflation, in which Planck time is

$$
t_{P}=\left(\hbar G c^{-5}\right)^{1 / 2} \sim 10^{-43} s
$$

The space originates the Planck length

$$
l_{P}=\left(\hbar G c^{-3}\right)^{1 / 2}=1.6 \times 10^{-33} \mathrm{~cm}
$$

Both already open a relation on unification of four interactions, in which G, c and $\hbar$ correspond respectively to gravitational, electromagnetic and microscopic strong-weak interactions. According to this model of the negative matter, inflation should end on a strong interaction scale $R_{e}=R_{i} e^{N} \approx 10^{-13} \mathrm{~cm}$, so $N=\ln \left(R_{e} / R_{i}\right)=20 \ln 10=46$. This is only $65 \%$ of now hypothesis $N=70$.

For $N=70=30 \ln 10$, the scale should extend to origin on $10^{-43} \mathrm{~cm}$ or end on $10^{-3} \mathrm{~cm}$, which corresponds to $V d W$ force and its extensive, and is consistent with phantom.

If the time is the current inflation time, it will be new interactions, such as weaker interactions at smaller scales, which also increase by only 2-3 orders of magnitude. Further, if the positive and negative matters may continually produce or annihilate for different positions and evolutional times of cosmos and galaxies, it will obtain the very complex relations between the positive and negative masses. This will have continual start of the Big Bang and the creation from nothing. Pairs of positive and negative matters correspond to pairs of super particles. For example, suppose the masses around the galaxy increase linearly with distance r, i.e., $M_{-}=a r$,

$$
v^{2}=\frac{G\left(M_{+}-M_{-}\right)}{r}=\frac{G M_{+}}{r}-G a \rightarrow G a=C
$$

Assume that $\left|m_{+}\right|=\left|m_{-}\right|$, so

$$
G_{\mu \nu}=R_{\mu \nu}-\frac{1}{2} g_{\mu \nu} R=8 \pi k\left(T_{\mu \nu}-T^{\prime}{ }_{\mu \nu}\right)=0
$$

This is not a vacuum, and is a case of complete opposite positive and negative matters. It seems to correspond to the origin of Big-Bang Universe.

\section{EVOLUTIONAL RATIO BETWEEN TOTAL MATTER AND USUAL MATTER}

The modern cosmology (Dodelson, 2003; Weinberg, 2008) includes an early inflation cosmology. Based on above mechanism of inflation, in a radiation-dominated universe of the big-bang cosmology, the usual total energy is mainly energy of photon $M_{+} c^{2}$. The total energy of positive and negative matters is

$$
M_{+} c^{2}-M_{-} c^{2}
$$

Because inflation is origin of nothing, the total energy should be zero, i.e., $M_{+}=M_{-}$.

When the evolutional process from inflation and radiationdominated universe to the matter-dominated universe, the known total energy of usual baryon matter of non-relativity is 


$$
M_{+} c^{2}-\frac{G M_{+}^{2}}{R}
$$

Assume that dark matter and dark energy are completely the negative matter, so the total energy includes three parts: one of the positive matter, one of negative matter, and their repulsion force

$$
E_{t}=M_{+} c^{2}-\frac{G M_{+}^{2}}{R}+\left(-M_{-} c^{2}-\frac{G M_{-}^{2}}{R}\right)+\frac{G M_{+} M_{-}}{R_{ \pm}}
$$

Both ratio is

$$
\frac{M_{+} c^{2}-\frac{G M_{+}^{2}}{R}+\left(-M_{-} c^{2}-\frac{G M_{-}^{2}}{R}\right)+\frac{G M_{+} M_{-}}{R_{ \pm}}}{M_{+} c^{2}-\frac{G M_{+}^{2}}{R}}
$$

We suppose that for early inflation cosmology the positive matter and the negative matter have the same mass $M_{+}=M_{-}=M$, and $R_{ \pm}=R$, so Eq.(58) is simplified to

$$
\frac{-\frac{G M^{2}}{R}}{M c^{2}-\frac{G M^{2}}{R}}=\frac{G M}{G M-R c^{2}}
$$

It is known that the total mass of Universe is $M=2 \times 10^{53} \mathrm{~kg}$, and corresponding scale is $R=4.2 G p c=1.3 \times 10^{26} \mathrm{~m}$ (Perkins, 2009), so

$$
R c^{2}=1.17 \times 10^{43} \mathrm{~m}^{3} \mathrm{~s}^{-2}
$$

and

$$
G M=1.34 \times 10^{43} \mathrm{~m}^{3} \mathrm{~s}^{-2}
$$

A simple calculation obtains $\frac{G M}{G M-R c^{2}}=7.88$.

If we take general ratio between usual matter, dark matter and dark energy is $4: 24: 72$, so ratio of total matter and the positive matter is $28 / 4=7$.

According to new data, usual matter is $4.84 \%$, total matter is $30.8 \%$, and dark energy is $69.2 \%$ (Adam et al., 2016; Tanabashi et al., 2018), so $30.8 / 4.84=6.36$. It is interesting evolutional result from $M_{+}=M_{-}$to 7.88 . If $R_{ \pm}=2 R$, Eq.(58) will be a simple formula

$$
\frac{3}{2} \frac{G M}{G M-R c^{2}}=11.82
$$

Conversely, for Eq.(59) let $\frac{G M}{G M-R c^{2}}=7$, we may determinate the quantitative relation between scale $\mathrm{R}$ and mass $M$ as $R=6 G M / c^{2}$. This should be a testable scale exhibited dark matter. If we take the galaxies mass $M=2 \times 10^{41} \mathrm{~kg}$, so $R=8.9 \times 10^{15} \mathrm{~m}$. It is about 1 light year $\left(9.46 \times 10^{15} \mathrm{~m}\right)$ or a fourth of $1 \mathrm{pc}$. For the solar system $M=2 \times 10^{30} \mathrm{~kg}$, so $R=8.9 \times 10^{4} \mathrm{~m}$, whose scale cannot condensate the negative matter, which must form conglomeration, and has the bigger scale. Such all structures (galaxies, stars, atoms, proton) dilacerated (Weinberg, 2008) are impossible.

\section{RELATIVITY AND THEORY OF LOBACHEVSKIAN GEOMETRY}

It is known that according to special relativity, the Lagrange function of any physical field should remain invariance under the Lorentz transformation (LT), which forms Lorentz transformation group. The general Lorentz group L has four leaves.

First leaf $\operatorname{det} \alpha=+1$ and $\alpha_{44} \geq 1$ is the proper LT group $L_{p}$. Second leaf $\operatorname{det} \alpha=-1$ and $\alpha_{44} \geq 1$ is the space inversion. First and second leaves compose the full Lorentz group $L_{f}$. Third leaf is $\operatorname{det} \alpha=-1$ and $\alpha_{44} \leq-1$.

Fourth leaf $\operatorname{det} \alpha=+1$ and $\alpha_{44} \leq-1$ is the total inversion $x_{\mu} \rightarrow-x_{\mu}$.

This is similar to four worlds (Fig.2) or their parts, in which $\operatorname{det} \alpha=+1$ and $\operatorname{det} \alpha=-1$ are topologically separated. They are similar to positive matter and negative matter. $\alpha_{44} \geq 1$ and $\alpha_{44} \leq-1$ are also topologically separated. Four leaves compose the extended Lorentz group $L_{e}$.

If first leaf corresponds to positive mass, and extends to general LT (GLT) (Chang, 1989, 2013a), it will be possibly related to positive-negative masses.

Third leaf is the time reflection. Since time corresponds to energy $\mathrm{E}$, mass $m=E / c^{2}$ is reflected to negative, i.e., $-m(c>0)$, or $m>0, c \rightarrow i c$, and $E<0$.

Existence of time inversion should correspond to mass-energy inversion, and various inversions of scalar, four-vector, tensor, torsion, rotation, etc. The particle number inversion corresponds to $\mathrm{C}$, and $\mathrm{C}$ corresponds to second and fourth worlds (Fig.2), and is related to PCT theorem.

Entropy $S=E / T$ corresponds to the irreversibility of time. It may be developed to four vectors, i.e., entropy flow

$$
S_{i}=\frac{a p_{i}}{T}=-i \hbar a \frac{\partial}{T \partial x_{i}}
$$

and

$$
S v=\frac{E v}{T}=\frac{m c^{2} v}{T}=\frac{p c^{2}}{T}=-i \hbar c^{2} \frac{\partial}{T \partial x}
$$

Further, it contacts Noether's theorem and its generalization (Chang, 2007a). When the Big Bang originated, the general energy must be not conserved, but all things is generated from nothing. Our theory is total energy conservation, but it extends to negative energy and the negative matter.

Space inversion corresponds to momentum $p=m v$ inversion, and it is $-v$ or $-m$. Space with $1>\alpha_{44}>-1$ and $\operatorname{det} \alpha \neq \pm 1$ should exist, which may be the space like interval, and associated with the light cone. The latter is more likely to be a curved space-time $\operatorname{det} \alpha=\left|g_{\mu v}\right| \neq \pm 1$.

Further, this may develop to Riemann space, general relativity and Lobachevsky space, etc. In Lobachevskian geometry plane $(\chi, \phi)$ (Dubrovin, Fomenko, \& Novikov, 1993), the trigonometric functions are replaced by the hyperbolic functions,

$$
\Gamma_{22}^{1}=-\frac{1}{2} \operatorname{sh}(2 \chi), \Gamma_{12}^{2}=\operatorname{cth} \chi
$$


Other, $\Gamma_{j k}^{i}=0$.

$$
\frac{d^{2} \chi}{d t^{2}}-\frac{1}{2} \operatorname{sh}(2 \chi)\left(\frac{d \phi}{d t}\right)^{2}=0, \frac{d^{2} \phi}{d t^{2}}+\operatorname{cth} \chi\left(\frac{d \phi}{d t} \frac{d \chi}{d t}\right)=0
$$

For space the metric $d s^{2}=g_{\mu v} d x_{\mu} d x_{v}, g_{\mu v}$ are all positive, it is Riemannian geometry; 1 or 2 in $g_{\mu v}$ are negative, it is Lobachevskian geometry, so both $\Gamma_{i j}^{k}$ are different. They are,

$$
\begin{aligned}
& d s^{2}=\frac{4 R^{4}}{\left(R^{2}+r^{2}\right)^{2}}\left(d r^{2}+r^{2} d \phi^{2}\right), R>0 \\
& d s^{2}=\frac{4 \alpha^{4}}{\left(\alpha^{2}-r^{2}\right)^{2}}\left(d r^{2}+r^{2} d \phi^{2}\right), R<0
\end{aligned}
$$

For the pseudo-sphere

$$
d s^{2}=d \rho^{2}-\rho^{2}\left(d \chi^{2}+\sinh ^{2} \chi d \phi^{2}\right)
$$

We may research possible special field equations for Lobachevskian geometry, and compare difference of scalar, vector, and tensor, etc., in both geometries.

These are possibly related to superstring. But, it is known from differential geometry that the curvature tensor is universal. Both difference is only the Gauss curvature $K=\frac{1}{r_{0}^{2}}>0$ and $K=-\frac{1}{r_{0}^{2}}<0$.

The Gaussian curvature is also

$$
K=-\frac{1}{2 g} \Delta \ln g=-\frac{1}{2} e^{-\phi} \Delta \phi
$$

It corresponds to $\Delta \phi=-2 K e^{\phi}$. If $K>0$, it is the sphere. If $K<$ 0 , it is the Lobachevskian plane. These are also related to the potential equations.

$$
\left(\frac{R^{\prime}}{R}\right)^{2}+\frac{k}{R^{2}}=\frac{8 \pi G}{3} \rho \quad(k=+1,0,-1)
$$

The metric on the sphere is (Dubrovin et al., 1993),

$$
d l^{2}=d r^{2}+\sin ^{2} \frac{r}{R_{0}} d \phi^{2} \quad(K>0)
$$

The metric on the Lobachevsian plane is

$$
d l^{2}=d r^{2}+\sinh ^{2} \frac{r}{R_{0}} d \phi^{2} \quad(K<0)
$$

From this we may overcome the singularity, and Lobachevskian geometry whose curvature $R<0$ may help to describe the mass-to-light ratio and the galactic rotational curves. According to the uncertainty principle, they correspond to very high momentum and energy,

$$
E=\hbar / t_{P}=6.58 \times 10^{-22} \mathrm{MeVs} / 5.39 \times 10^{-44} \mathrm{~s}=1.22 \times 10^{22} \mathrm{MeV}
$$

and

$$
P=\hbar / l_{P}=6.58 \times 10^{-22} \mathrm{MeVs} / 1.62 \times 10^{-35} \mathrm{~m}=4.06 \times 10^{13} \mathrm{MeVs} / \mathrm{m} .
$$

Based on both we obtain the positive matter and negative matter created from nothing at the same time, and form twoworlds (Chang, 2007b, 2013b, 2017). Inflation is from Planck time-space to strong interaction scaling $10^{-13} \mathrm{~cm}$ and corresponding time $10^{-23} \mathrm{~s}$. The very early strong interaction must be derived from quantum fluctuations, and obtained the non-uniformity.

Dark matter particles are the product of the early very high temperature in the Big Bang. It is consistent completely with creation of the negative matter. In the very early inflation the total energy with strong repulsion is

$$
E=M_{+} c^{2}-M_{-} c^{2}+\frac{g M_{+} M_{-}}{R_{ \pm}} e^{-M r}
$$

Assume that $\left|m_{+}\right|=\left|m_{-}\right|, \quad F=\frac{g|M|^{2}}{R_{ \pm}{ }^{2}} e^{-M r} \quad$ is namely the exponential expansion in universe.

In inflation the invariant vacuum energy is the initial energy generated simultaneously by the positive and negative matters, which is conservation $E=2 E_{\text {Planck }}=2.44 \times 10^{19} \mathrm{GeV}$, and forms two or four worlds (Fig.2), which are different universes and multiverse.

Further, based on the Big Bang cosmology we may develop a cosmologic projective geometry unified various nonEuclidean geometries discussed by F.Klein and A. Cayler.

Usual positive matter and negative matter repulsion each other. But, if charge and electromagnetic interaction exist, there will have gravitation.

First we suppose that positive matter and negative matter are separated, and then both split into positive and opposite matters, and create charge. Assume that matter particles have $\mathrm{N}$, and a part in $\mathrm{N}$ creates charge $\alpha N Q$, in which half positive, and half negative, i.e., $\alpha N Q / 2$. Let $\left|m_{+}\right|=\left|m_{-}\right|$, and $\left|Q_{+}\right|=|Q|=Q$, so a total force is

$$
F_{t}=F_{m}+F_{Q}=\frac{G|m|^{2}}{r^{2}}-\frac{(\alpha N|Q|)^{2}}{4 r^{2}}
$$

When $\sqrt{G} m<(\alpha N Q / 2)$, i.e., the stronger electromagnetic interaction exists, this is gravitational force. It derives probably the opposite matter decreased more.

After the 1990s, the observations of the cosmic microwave background radiation (CMB) anisotropy by COBE, etc., are consistent with the prediction of inflation, and exclude basically the topological defects as the main source of initial disturbance. 
JUDGMENT TEST OF NEGATIVE MATTER AS DARK MATTER AND OTHER EIGHT TESTS

Based on observations of a remarkable cosmic structure called the bullet cluster, Clowe et al. (2006) discovered that this structure is actually two clusters of galaxies passing through one another. Past observations have shown that only a very small percentage of mass in the universe can be explained by regular matter. The new research is the first to detect luminous matter and dark matter independent of one another, with the luminous matter clumped together in one region and the dark matter clumped together in another. These observations demonstrate that there are two types of matter: one visible and one invisible.

In 2007, COSMOS obtained first three-dimensional distribution map of dark matter on world (Massey et al., 2007). In May 2011 Atacama cosmology telescope found the trace of dark energy in the cosmic microwave background (Sherwin et al., 2011), and in which found the weak lens phenomenon (CMB lensing) (Das et al., 2011).

The negative matter is completely symmetry with positive matter, and it as dark matter should agree with the principle of cosmology and is uniform on a large scale. But, it is groups, so it is impossible that the whole universe is full of dark matter. General relativity is very precise in the solar system, in which there cannot have dark energy. Various positive matter and black hole exhibit the gravitational lensing effect. While the negative matter and negative black hole will be the repulsive lensing phenomena. Both should be different in observations. For the negative matter there should also have the corresponding black hole, whose radius is

$$
r=-2 G m / c^{2}
$$

It is known that celestial body with huge massive matter has the gravitational lensing effect (Fig.3)(Cai, Saridakis, Setare, \& Xia, 2010; Chae et al., 2002; Copeland, Sami, \& Tsujikawa, 2006).

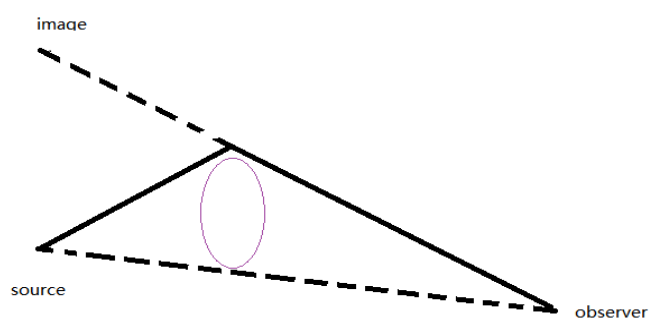

Fig.3. Gravitational lensing

Since usual light under interaction of negative matter is repulsive deflection, so it will show the repulsive lensing effect.

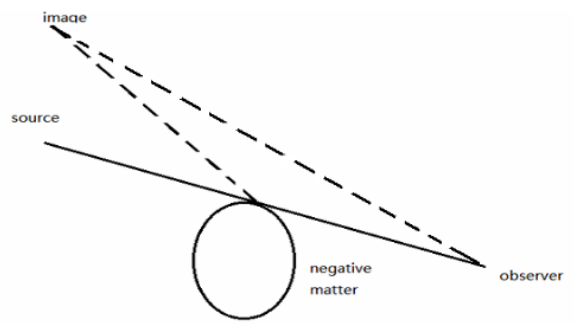

Fig.4. Repulsive lensing
We proposed a judgment test for the negative matter as dark matter (Chang, 2019): This is an opposite repulsive lensing (Fig.4). We calculate a deflected angle derived from the negative matter. Based on the field equations we may similarly obtain the formula

$$
\Delta \phi=-\frac{4 G M}{R c^{2}}
$$

$\mathrm{M}$ is bigger, so is also $\Delta \phi$. Therefore, the lensing has three kinds.

1-Visible celestial body with large mass, (gravitation deflection).

2-Invisible black hole with large mass, (gravitation deflection)

3-Invisible negative matter with large mass, (repulsion deflection).

Mass of the negative matter is invariant, and mass of black hole may increase and form an accretion disk. Further, from (78) we may determinate three kinds of mass on the negative matter and general matter

$$
M_{ \pm}= \pm \frac{R c^{2}}{4 G} \Delta \phi
$$

Microlensing Observations in Astrophysics team and Optical Gravitational Lensing Experiment team (Soszynski et al., 2004) have searched widely for dark stars and exoplanets by microlensing. Their results should provide possible data. The evolution of the negative matter may form corresponding white dwarfs, neutron stars and black holes of the negative matter. They will produce the repulsive lensing.

At present, JA Giannini (2019) proposed that developed the field equations (Chang, 2007b, 2013b) describing the repulsive interaction, which is another possibility being explored is the repulsive force of negative mass and its potential relation to dark matter in unified positive and negative mass sources all scales. Judith Giannini (2019), researched fractal composite quarks and leptons with positive and negative mass components.

The numerical simulations of cold dark matter predicted that there are many dark matter halos (Fig.5).

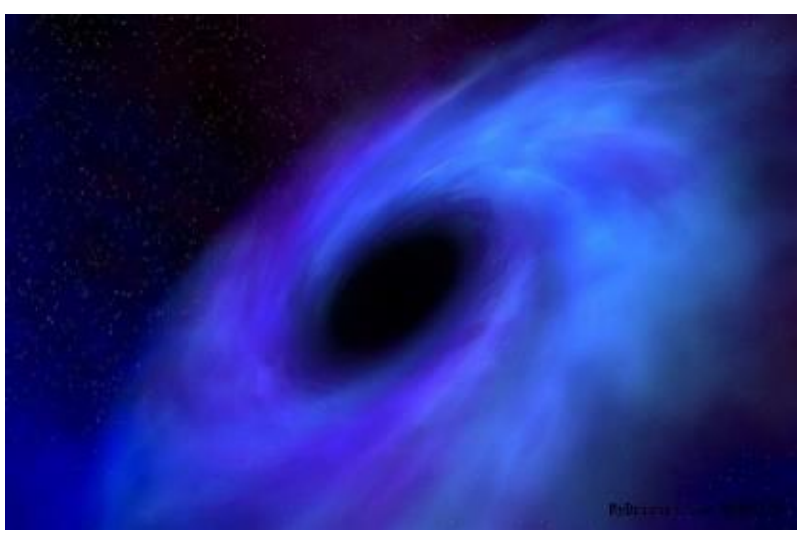

Fig.5. Dark matter as the Galactic halo

Gentile, Famaey, Zhao, and Salucci (2009), discussed universality of galactic surface densities within one dark halo scale-length. These halos around the galaxy are expected to 
observe many satellite galaxies (Kravtsov, 2010). But, the actual observation of satellite galaxies is far less than theoretical predictions. Assume that the halo of negative-dark matter forms the spherical shell distribution, whose mass is

$$
\begin{gathered}
m_{-}=\rho d S=4 \pi \rho\left(r_{0}+d r\right)^{2} \\
d v=\frac{F}{M} d t=-G \frac{M\left(m_{+}-m_{-}\right)}{M r^{2}} d t
\end{gathered}
$$

When $r=r_{0}, m_{+} \rightarrow 0$, so

$$
d v=G \frac{m_{-}}{r_{0}^{2}} d t=4 \pi \rho G\left(1+\frac{d r}{r_{0}}\right)^{2} d t
$$

This as a constant agrees with the mass-to-light ratio of galaxies. Generally, we propose other eight possible tests of this hypothesis:

(1) In a large scale space, if there is a negative mass cluster in positive matter, some positive matter will be screened, and the other visible matter will be changed by repulsion lensing. As a result, visible matter appears less, and the negative matter and its screened positive matter will appear as invisible dark matter. According to this assumption, the visible celestial phenomena and dark matter will be slightly different because the screened and distorted parts are different for those observations from different positions of the Earth in the solar system. The season effects of this dark matter are observable.

(2) There is repulsive force between positive and negative matters, and which obey the square inverse ratio repulsive law according to the Newtonian law. This agrees with the shape of dark matter as the Galactic halo (Rita Bernabei et al., 2013; Chang, 2016; Gentile et al., 2009).

(3) General photons are reflected by the negative matter, which exhibits a type of dark matter.

(4) When the move speeds between positive and negative matters are very big, and the kinetic energy is bigger than the potential energy, their colliding result will be a complete annihilation, whose leftover is only a mass-difference of positive and negative matters. These may indicate very high energy phenomena in astronomy.

(5) The negative matter may represent the cosmic repulsion and the fast expansion.

(6) The positive and negative matters under some exceeding conditions may be created from nothing to all thing at the same time, both are a huge repulsion and rapid expansion, which derives the inflation universe (Chang, 2007b, 2013b, 2014a, 2016, 2019).

(7) It must exist that some repulsion field regions obey Lobachevskian geometry with curvature $R<0$.

(8) The negative matter is similar to invisible black hole, but is repulsive force for matter, and its mass is invariant.
In these tests the season effect may explain DAMA observed the annual modulation $8.9 \sigma$ (R Bernabei et al., 2008; R Bernabei et al., 2000).

DAMA/LIBRA (Rita Bernabei et al., 2013) obtains a final model independent result of DAMA/LIBRA-phase. CoGeNT collaboration observed possible signs of light dark matter particles, and had similar annual modulation (Aalseth, Barbeau, Bowden, et al., 2011; Aalseth, Barbeau, Colaresi, et al., 2011) But, CDMS experiments measured electronic and phonon signals caused by dark matter scattering (Ahmed \& collaboration, 2009), and XENON100 experiments detected luminous and ionizing signals after dark matter scattering (Aprile et al., 2011), both were not found DAMA results. Probably, dark matter does not generate these signals.

\section{COMPLETE WORLD AND UNIFICATION OF FOUR INTERACTIONS}

Mass $\mathrm{M}$ and charge $\mathrm{Q}$ are the most important matter quantities. Opposite $\mathrm{Q}$ is anti-(opposite) matter, and opposite $\mathrm{M}$ is the negative matter. A stronger electromagnetic interaction between opposite matter and negative matter could lead to a significant decrease in opposite matter.

In M-Q plane $z=m+i Q=\rho(\cos \phi+i \sin \phi)$, modular $\rho=\sqrt{m^{2}+Q^{2}}>0$. The negative matter with electromagnetic field is

$$
E^{2}=m^{2} c^{4}+c^{2}\left(p-\frac{e}{c} A\right)^{2}
$$

For $m=0$, energy is $E= \pm c\left(p-\frac{e}{c} A\right)$ and for $E=0$, then $m^{2}=-\frac{1}{c^{2}}\left(p-\frac{e}{c} A\right)^{2}<0$. It is known that positive and negative matters are repulsion each other, but because charges exist between various matters, so they can also be attraction.

When $Q_{+} Q_{-}>G M_{+} M_{-}$, from this may produce the big mass annihilation, in which the charge is partially similar $e^{+} e^{-} \rightarrow \gamma$. Therefore, the stable particles of dark matter are mainly neutral charge. Moreover, the attraction of only a few electromagnetic interactions compared with the repulsive force determined by huge mass may probably be presented as weakly interacting massive particles (WIMPs). This is also related to the quantum gravity theory, and superstring, supergravity, etc.

This is not only a unified gravitational field and electromagnetic field, but a unified four types of matter. In the complete world with four perfect symmetries (Fig.2), the lower symmetry of positive matter world is the supersymmetry of fermions and bosons and their breaking. Fermions are more specific to quark-lepton symmetry and three-generation symmetry, in which symmetry breaks, such as different isospin I. We think spin of particle of repulsion (repulsion) is possibly $J=-2$. If repulsion exists, there will be particles $J=$ $2,1,0,-1,-2$, which may be unified bosons (graviton, photon, meson and so on).

The unification of various interactions is always an important question in physics. Strong and weak interactions are all shortrange, which should more be unified. It is known that the strong interactions are attraction each other, and the weak interactions are mutual repulsions and derive decay. We proposed both "running" coupling constants transform each other following distances and energy, and pass through the asymptotic freedom of QCD. They correspond to the coupling 
constant $G>0$ and $G<0$. The two aspects are respectively QCD with SU (3) and QWD (quantum weak dynamics) with SU (2), and may be unified by the Yang-Mills gauge field, and between them is asymptotically free $G=0$. We research some possible tests and predictions, which are consistent with the experiments on the quark-parton model, and various scatterings from Pomeranchuk theorem to the cross sections at higher energy gradual increase. When strong and weak interactions change, and both transform each other, they may produce different waves, for example, neutrino wave for weak interaction. These particle waves possess the uncertainty relation (Chang, 1989, 2018b), and are generally $m \neq 0$ and $v<c$, and are the not-Abel gauge fields and the nonlinear short-range waves. But, according to the de Broglie relation $v \bar{v}=c^{2}$, the particle velocity $\mathrm{v}$ is related to the phase velocity $\bar{v}>c$ that obeys the general Lorentz transformation (GLT)(Chang, 1989, 2013a), and corresponds to the quantum entangled state (Chang, 2013a).

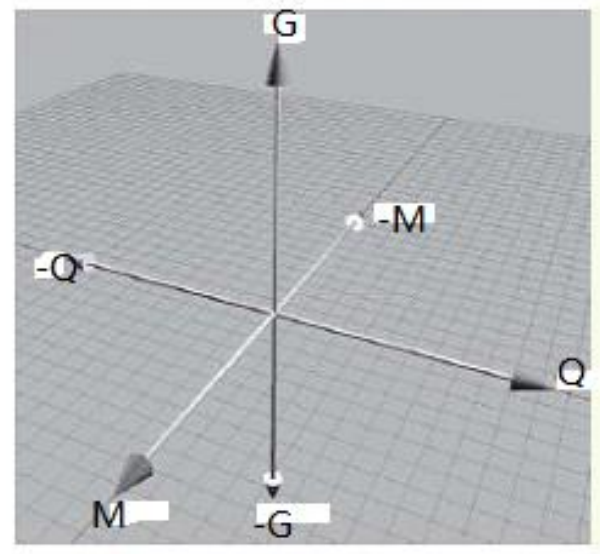

Fig. 6 The unification on the four basic interactions in threedimensional space.

\section{REFERENCES}

Aalseth, C. E., Barbeau, P., Bowden, N., Cabrera-Palmer, B., Colaresi, J., Collar, J., .Fields, N. (2011). Results from a search for light-mass dark matter with a p-type point contact germanium detector. Physical Review Letters, 106(13), 131301.

Aalseth, C. E., Barbeau, P., Colaresi, J., Collar, J., Leon, J. D., Fast, J. E., . . Kephart, J. D. (2011). Search for an annual modulation in a p-type point contact germanium dark matter detector. Physical Review Letters, 107(14), 141301.

Abbott, B. P., Abbott, R., Abbott, T., Abernathy, M., Acernese, F., Ackley, K., . . . Adhikari, R. (2016a). GW150914: Implications for the stochastic gravitationalwave background from binary black holes. Physical Review Letters, 116(13), 131102.

Abbott, B. P., Abbott, R., Abbott, T., Abernathy, M., Acernese, F., Ackley, K., . . Adhikari, R. (2016b). Observation of gravitational waves from a binary black hole merger. Physical Review Letters, 116(6), 061102.
We proposed the most perfect symmetrical world (Fig.2). As a two-dimensional plane this also corresponds to gravitational and electromagnetic fields determined by mass and charge, respectively. Further, we combine the four known fundamental interactions to develop into the four-dimensional space. But in some respect, this can be simplified to threedimensional space, where the third dimension is assumed to be the strong and weak interactions of the short-range. Strong interaction $G>0$ is the upper sides, and weak interaction $G<$ 0 is the lower side, respectively, so that Fig. 6 can uniformly describe the four basic interactions (Chang, 2020).

\section{DISCUSSION}

Morris and Thorne discussed wormhole, and exotic matter has negative pressure and opposite gravitation, which can maintain an open macroscopic wormhole (Morris, Thorne, \& Yurtsever, 1988). If the negative matter is exotic matter, it will able to maintain an open macro wormhole. The wormhole of the negative matter is just the opposite gravitation, i.e., repulsion. The negative matter has also black hole. But, between positive and negative matters probably is the white hole. The collision of groups of positive-negative matters, on the one hand, is rapidly separated since repulsion; on the other hand, very few of them will be annihilated, which may also be accompanied by high-energy phenomena. If dark matter and dark energy exist, the critical density will increase by 6-7 times and 25 times. In this case, the universe will contract, cycle, etc.

The negative matter as a candidate of unification of dark matter and dark energy is not only the simplest, and may explain inflation and be calculated and tested. It agrees on Occam's Razor. But, some hypotheses on dark matter and dark energy are not testability in epistemology. Moreover, early in 1898 Schuster (1898) conjectured the existence of new sun with the negative mass.

Abbott, B. P., Abbott, R., Abbott, T., Acernese, F., Ackley, K., Adams, C., . . Adya, V. (2017). GW170817: observation of gravitational waves from a binary neutron star inspiral. Physical Review Letters, 119(16), 161101.

Adam, R., Ade, P. A., Aghanim, N., Akrami, Y., Alves, M., Argüeso, F., . . A Aumont, J. (2016). Planck 2015 results-I. Overview of products and scientific results. Astronomy \& Astrophysics, 594, A1.

Ahmed, Z., \& collaboration, C. (2009). Results from the Final Exposure of the CDMS II Experiment. arXiv preprint arXiv:0912.3592.

Albrecht, A., \& Steinhardt, P. J. (1982). Cosmology for grand unified theories with radiatively induced symmetry breaking. Physical Review Letters, 48(17), 1220.

Aprile, E., Arisaka, K., Arneodo, F., Askin, A., Baudis, L., Behrens, A., . . Bruno, G. (2011). Dark matter results from 100 live days of XENON100 data. Physical Review Letters, 107(13), 131302.

Ballentine, L. (1998). Quantum mechanics-a modern development-L. Ballentine. Am. J. Phys, 59, 1153. 
Barger, V., Langacker, P., McCaskey, M., Ramsey-Musolf, M. J., \& Shaughnessy, G. (2008). CERN LHC phenomenology of an extended standard model with a real scalar singlet. Physical Review D, 77(3), 035005.

Bernabei, R., Belli, P., Cappella, F., Caracciolo, V., Castellano, S., Cerulli, R., Di Marco, A. (2013). Final model independent result of DAMA/LIBRA-phase1. The European Physical Journal C, 73(12), 2648.

Bernabei, R., Belli, P., Cappella, F., Cerulli, R., Dai, C., d'Angelo, A., ... Ma, J. (2008). First results from DAMA/LIBRA and the combined results with DAMA/NaI. The European Physical Journal C, 56(3), 333-355.

Bernabei, R., Belli, P., Cerulli, R., Montecchia, F., Amato, M., Ignesti, G., . . He, H. (2000). Search for WIMP annual modulation signature: Results from DAMA/NaI-3 and DAMA/NaI-4 and the global combined analysis. Physics Letters B, 480(1-2), 23-31.

Binney, J., \& Tremaine, S. (2011). Galactic dynamics: Princeton university press.

Bondi, H. (1957). Negative mass in general relativity. Reviews of modern physics, 29(3), 423.

Cai, Y.-F., Saridakis, E. N., Setare, M. R., \& Xia, J.-Q. (2010). Quintom cosmology: theoretical implications and observations. Physics Reports, 493(1), 1-60.

Caldwell, R. R. (2002). A phantom menace? Cosmological consequences of a dark energy component with supernegative equation of state. Physics Letters B, 545(1-2), 2329.

Chae, K.-H., Biggs, A., Blandford, R., Browne, I., De Bruyn, A., Fassnacht, C., Koopmans, L. (2002). Constraints on cosmological parameters from the analysis of the Cosmic Lens All Sky Survey radio-selected gravitational lens statistics. Physical Review Letters, 89(15), 151301.

Chang, Y.-F. (1989). New Research of Particle Physics and Relativity. Yunnan Science and Technology Press. Phys.Abst.93,1371.

Chang, Y.-F. (1990). Development of Titius-Bode rule and cosmic quantum theory. $\mathrm{PBeiO}, 16,16$.

Chang, Y.-F. (1996). Nonlinear nature of gravitational waves. Apeiron, 3(2), 30-32.

Chang, Y.-F. (2002). Development of the Titius-Bode Law and the Extensive Quantum Theory. Physics Essays, 15(2).

Chang, Y.-F. (2005). GRT extended for electromagnetic fields: equivalence principle and geometrization. Galilean Electrodynamics, 16(5), 91.

Chang, Y.-F. (2007a). Fractal Relativity, Generalized Noether Theorem and New Research of Space-Time. arXiv preprint arXiv:0707.0136.

Chang, Y.-F. (2007b). Negative matter, repulsion force, dark matter and inflation cosmos, Higgs mechanism. arXiv:0705.2908.

Chang, Y.-F. (2011). Negative matter, dark matter and theoretical test. International Review of Physics, 5(6), 340345 .
Chang, Y.-F. (2013a). Extension and complete structure of the special relativity included superluminal and neutrinophoton with mass. International Journal of Modern Theoretical Physics, 2(2), 53-73.

Chang, Y.-F. (2013b). Field Equations of Repulsion Force between Positive-Negative Matter, Inflation Cosmos and Many Worlds. Int. J. Mod. Theoretical Phys, 2, 100-117.

Chang, Y.-F. (2014a). Astronomy, black hole and cosmology on negative matter, and qualitative analysis theory. Int. J. Mod. Applied Phys, 4(2), 69-82.

Chang, Y.-F. (2014b). Unified theories of gravitational and electromagnetic fields in Riemannian geometry and higher dimension. International Review of Physics. 8,5,132-140., 132.

Chang, Y.-F. (2016). Calabi-Yau Manifolds of NebulaGalaxies and Their Possible Dynamics, and Gravitational Wave. International Journal of Modern Theoretical Physics. 5,1,22-38.

Chang, Y.-F. (2017). Negative matter as unified dark matter and dark energy, and possible tests. Hadronic J, 40(3), 291308.

Chang, Y.-F. (2018a). Basic Principles of Physics and Their Applications, and Logical Structure of Quantum Mechanics. International Journal of Theoretical Physics. 7,1,16-39.

Chang, Y.-F. (2018b). Extensive quantum theory with different quantum constants, and its applications. International Journal of Modern Mathematical Sciences, 16(2), 148-164.

Chang, Y.-F. (2019). Negative matter as dark matter, and its judgment test and calculation of ratio. International Journal of Modern Applied Physics, 9(1), 1-12.

Chang, Y.-F. (2020). Unification of strong-weak interactions and possible unified scheme of four-interactions. European Journal of Applied Sciences. 8,5, 28-45.

Chevallier, M., \& Polarski, D. (2001). Accelerating universes with scaling dark matter. International Journal of Modern Physics D, 10(02), 213-223.

Chimento, L. P., Forte, M., Lazkoz, R., \& Richarte, M. G. (2009). Internal space structure generalization of the quintom cosmological scenario. Physical Review D, 79(4), 043502.

Clowe, D., Bradač, M., Gonzalez, A. H., Markevitch, M., Randall, S. W., Jones, C., \& Zaritsky, D. (2006). A direct empirical proof of the existence of dark matter. The Astrophysical Journal Letters, 648(2), L109.

Copeland, E. J., Sami, M., \& Tsujikawa, S. (2006). Dynamics of dark energy. International Journal of Modern Physics D, 15(11), 1753-1935.

Copi, C. J., Schramm, D. N., \& Turner, M. S. (1995). Bigbang nucleosynthesis and the baryon density of the universe. Science, 267(5195), 192-199.

Das, S., Sherwin, B. D., Aguirre, P., Appel, J. W., Bond, J. R., Carvalho, C. S., Essinger-Hileman, T. (2011). Detection of the power spectrum of cosmic microwave background 
lensing by the atacama cosmology telescope. Physical Review Letters, 107(2), 021301.

Dirac, P. (1958). The principles of quantum mechanics, Oxford Univ. Press, London.

Dirac, P. A. M. (1930). A theory of electrons and protons. Proceedings of the Royal Society of London. Series A, Containing papers of a mathematical and physical character, 126(801), 360-365.

Dodelson, S. (2003). Modern cosmology: Elsevier.

Dodelson, S., Kinney, W. H., \& Kolb, E. W. (1997). Cosmic microwave background measurements can discriminate among inflation models. Physical Review D, 56(6), 3207.

Dubrovin, B., Fomenko, A., \& Novikov, S. (1993). Modern geometry-Methods and applications. Part I," 0¥ edition: Springer Verlag, New York.

Einstein, A. (1955). the meaning of Relativity (fifth edi.): Princeton niversity Press, Princeton, NJ.

Elahi, F., \& Khatibi, S. (2019). Multi-component dark matter in a non-Abelian dark sector. Physical Review D, 100(1), 015019.

Feng, C.-J., Li, X.-Z., \& Saridakis, E. N. (2010). Preventing eternality in phantom inflation. Physical Review D, 82(2), 023526.

Flügge, S. (2012). Practical quantum mechanics: Springer Science \& Business Media.

Gentile, G., Famaey, B., Zhao, H., \& Salucci, P. (2009). Universality of galactic surface densities within one dark halo scale-length. Nature, 461(7264), 627-628.

Gerhard, O., \& Silk, J. (1996). Baryonic dark halos: A cold gas component? The Astrophysical Journal, 472(1), 34.

Giannini, J. (2019). Feasibility of Constructing a Unified Positive and Negative Mass Potential. Int. J. Mod. Theoretical Phys, 8, 1-16.

Giannini, J. (2019). Fractal Composite Quarks and Leptons with Positive and Negative Mass Components. Int. J. Modern Theo. Physics, 8(1), 41-63.

Gonzalez, J., \& Guzman, F. (2009). Accretion of phantom scalar field into a black hole. Physical Review D, 79(12), 121501.

Guth, A. H. (1981). Inflationary universe: A possible solution to the horizon and flatness problems. Physical Review D, 23(2), 347.

Hong, S.-T., Lee, J., Lee, T. H., \& Oh, P. (2008). Higher dimensional cosmological model with a phantom field. Physical Review D, 78(4), 047503.

Hooper, D., \& Profumo, S. (2007). Dark matter and collider phenomenology of universal extra dimensions. Physics Reports, 453(2-4), 29-115.

Jungman, G., Kamionkowski, M., \& Griest, K. (1996). Supersymmetric dark matter. Physics Reports, 267(5-6), 195-373.

Kahn, F., \& Woltjer, L. (1959). Intergalactic Matter and the Galaxy. The Astrophysical Journal, 130, 705.
Kim, J. H., Lane, S. D., Lee, H.-S., Lewis, I. M., \& Sullivan, M. (2020). Searching for dark photons with maverick top partners. Physical Review D, 101(3), 035041.

Kravtsov, A. (2010). Advances in Astronomy 2010, 281913 (2010). arXiv preprint arXiv:0906.3295.

La, D., \& Steinhardt, P. J. (1989). Erratum:“'Extended inflationary cosmology"[Phys. Rev. Lett. 62, 376 (1989)]. PhRvL, 62(9), 1066.

Landau, L., \& Lifshitz, E. (1977). Quantum Mechanics (Nonrelativistic theory): Oxford: Pergamon Press.

Landau, L. D., \& Lifshitz, E. M. (1971). The classical theory of fields.

Linde, A. (1994). Hybrid inflation. Physical Review D, 49(2), 748.

Linde, A. D. (1982). A new inflationary universe scenario: a possible solution of the horizon, flatness, homogeneity, isotropy and primordial monopole problems. Physics Letters B, 108(6), 389-393.

Linde, A. D. (1983). Chaotic inflation. Physics Letters B, 129(3-4), 177-181.

Lucchin, F., \& Matarrese, S. (1985). Power-law inflation. Physical Review D, 32(6), 1316.

Mannheim, P. D. (1992). Conformal gravity and the flatness problem. The Astrophysical Journal, 391, 429-432.

Massey, R., Rhodes, J., Ellis, R., Scoville, N., Leauthaud, A., Finoguenov, A., Kneib, J.-P. (2007). Dark matter maps reveal cosmic scaffolding. Nature, 445(7125), 286-290.

McDonald, J. (1994). Gauge singlet scalars as cold dark matter. Physical Review D, 50(6), 3637.

Morris, M. S., Thorne, K. S., \& Yurtsever, U. (1988). Wormholes, time machines, and the weak energy condition. Physical Review Letters, 61(13), 1446.

Mortonson, M. J., Hu, W., \& Huterer, D. (2010). Testable dark energy predictions from current data. Physical Review D, 81(6), 063007.

Peebles, P. J. E., \& Ratra, B. (2003). The cosmological constant and dark energy. Reviews of modern physics, 75(2), 559

Perkins, D. H. (2009). Particle astrophysics: Oxford University Press.

Perlmutter, S., Aldering, G., Della Valle, M., Deustua, S., Ellis, R., Fabbro, S., . . Hook, I. (1998). Discovery of a supernova explosion at half the age of the Universe. Nature, 391(6662), 51-54.

Piao, Y.-S., \& Zhang, Y.-Z. (2004). Phantom inflation and primordial perturbation spectrum. Physical Review $D$, 70(6), 063513.

Riess, A. G., Filippenko, A. V., Challis, P., Clocchiatti, A., Diercks, A., Garnavich, P. M., . . Kirshner, R. P. (1998). Observational evidence from supernovae for an accelerating universe and a cosmological constant. The Astronomical Journal, 116(3), 1009.

Russo, J. G. (2004). Exact solution of scalar field cosmology with exponential potentials and transient acceleration. Physics Letters B, 600(3-4), 185-190. 
Saridakis, E. N., \& Sushkov, S. V. (2010). Quintessence and phantom cosmology with nonminimal derivative coupling. Physical Review D, 81(8), 083510.

Scherrer, R. J. (2004). Purely kinetic k essence as unified dark matter. Physical Review Letters, 93(1), 011301.

Scherrer, R. J., \& Sen, A. (2008). Phantom dark energy models with a nearly flat potential. Physical Review D, $78(6), 067303$.

Schuster, A. (1898). Potential Matter.-A Holiday Dream. Nature, 58(1503), 367-367.

Shellard, E., \& Battye, R. (1998). Origin of dark matter axions. Physics Reports, 307(1-4), 227-234.

Sherwin, B. D., Dunkley, J., Das, S., Appel, J. W., Bond, J. R., Carvalho, C. S., . Fowler, J. W. (2011). Evidence for dark energy from the cosmic microwave background alone using the Atacama Cosmology Telescope lensing measurements. Physical Review Letters, 107(2), 021302.
Soleng, H. H. (1995). Dark matter and non-newtonian gravity from general relativity coupled to a fluid of strings. General Relativity and Gravitation, 27(4), 367-378.

Soszynski, I., Udalski, A., Kubiak, M., Szymanski, M., Pietrzynski, G., Zebrun, K., Wyrzykowski, L. (2004). The Optical Gravitational Lensing Experiment. Small Amplitude Variable Red Giants in the Magellanic Clouds. arXiv preprint astro-ph/0407057.

Tanabashi, M., Hagiwara, K., Hikasa, K., Nakamura, K., Sumino, Y., Takahashi, F., Amsler, C. (2018). Review of particle physics. Physical Review D, 98(3), 030001.

Weinberg, S. (1989). The cosmological constant problem. Reviews of modern physics, 61(1), 1.

Weinberg, S. (2008). Cosmology: Oxford university press. Zwicky, F. (1937). On the Masses of Nebulae and of Clusters of Nebulae. The Astrophysical Journal, 86, 217. 